\title{
SUPERCONDUCTING AND MAGNETIC PHASES WITH 1212-TYPE OF STRUCTURE
}

\author{
E.P. KhLYbov ${ }^{a, b}$, I.E. Kostyleva ${ }^{b}$, K. NenKov ${ }^{a, c}$, V.I. NizhankovsKiI ${ }^{a}$, \\ T. PALEWSKI ${ }^{a}$ AND J. WARCHULSKA ${ }^{a}$ \\ a International Laboratory of High Magnetic Fields and Low Temperatures \\ Gajowicka 95, 53-421, Wrocław, Poland \\ ${ }^{b}$ Institute of High Pressure Physics, 142092 Troitsk Moscow reg, Russia \\ ${ }^{c}$ Institut fur Festkörper- und Werkstofforschung Dresden \\ P.O.Box 270016, 01171 Dresden; Germany
}

Studies of magnetic and transport properties of the 1212 compounds $\mathrm{MA}_{2}^{\prime} \mathrm{A}^{\prime \prime} \mathrm{Cu}_{2} \mathrm{O}_{7-x}\left(\mathrm{M}=\mathrm{Al}\right.$ or $\mathrm{Tl} ; \mathrm{A}^{\prime}=\mathrm{Sr}$ or $\mathrm{Ba} ; \mathrm{A}^{\prime \prime}=\mathrm{RE}, \mathrm{Y}$, and $\mathrm{Ca}$ ) revealed a number of interesting magnetic phenomena. Apart from high-temperature superconductivity, these compounds exhibit spontaneous antiferromagnetism, field induced antiferromagnetism or ferromagnetism. The different magnetic orders and superconductivity may be realized in the same compound as a result of the phase separation.

PACS numbers: $74.25 . \mathrm{Ha}, \mathbf{7 4 . 7 2 . J t}$

\section{Introduction}

In last years the perovskite-like compounds have attracted a great deal of interest in many laboratories because of a wide range of interesting physical properties both superconducting and magnetic. Besides high-temperature superconductors (HTSC) they include compounds revealing different types of magnetic ordering. These circumstances open a promising and exciting possibility to study the interaction between so different types of order parameters. From this point of view the cuprates with general formulae $\mathrm{MA}_{2}^{\prime} \mathrm{A}^{\prime \prime} \mathrm{Cu}_{2} \mathrm{O}_{7-x}\left(\mathrm{M}=\mathrm{Al}, \mathrm{Tl} ; \mathrm{A}^{\prime}=\right.$ $\mathrm{Sr}, \mathrm{Ba} ; \mathrm{A}^{\prime \prime}=\mathrm{RE}, \mathrm{Y}$, and $\mathrm{Ca}$ ) crystallizing in the so-called 1212-type of structure may supply a great variety of experimental data because they show bright superconducting properties $\left(\mathrm{TlBa}_{2} \mathrm{CaCu}_{2} \mathrm{O}_{7-x}\right.$ has $\left.T_{\mathrm{c}}=110 \mathrm{~K}\right)$ as well as interesting magnetic ones. The coexistence of superconductivity and magnetic ordering is the subject of especial interest for researchers. Moreover, the 1212 compounds are promising materials for such investigations because both impurity phase separation and electronic phase separation can occur there.

\section{Experimental}

Polycrystalline samples of $\mathrm{MA}_{2}^{\prime} \mathrm{A}^{\prime \prime} \mathrm{Cu}_{2} \mathrm{O}_{7-x}\left(\mathrm{M}=\mathrm{Al}, \mathrm{Tl} ; \mathrm{A}^{\prime}=\mathrm{Sr}, \mathrm{Ba} ; \mathrm{A}^{\prime \prime}=\right.$ $\mathrm{RE}, \mathrm{Y}$, and $\mathrm{Ca}$ ) compounds with a typical for HTSC 1212-type structure have 
been synthesized and investigated. Samples for the investigations were prepared by a solid state reaction at temperatures of $840-1080^{\circ} \mathrm{C}$ for $4-24$ hours using well-homogenized mixtures of $\mathrm{M}_{2} \mathrm{O}_{3}, \mathrm{R}_{2} \mathrm{O}_{3}, \mathrm{CuO}$ oxides, and $\mathrm{SrCO}_{3}$ or $\mathrm{BaCO}_{3}$ carbonates. If necessary the samples were additionally milled and several times annealed to obtain a single-phase composition. The X-ray diffraction analysis was carried out on a Geigerflex diffractometer D-2 using $\mathrm{Cu} K_{\alpha}$ radiation and a bent graphite monochromator. Some X-ray diffraction patterns were taken using an automated Phillips PW 1820 diffractometer with $\mathrm{Co} K_{\alpha}$ radiation. X-ray diffraction measurements indicate that all samples are single phase 1212-type material. The crystal structure of the $\mathrm{AlSr}_{2} \mathrm{RCu}_{2} \mathrm{O}_{7-x}(\mathrm{R}=\mathrm{Y}, \mathrm{Er}, \mathrm{Ho})$ compounds was additionally studied using neutron diffraction measurements [1]. Note that the oxygen content in the Al-containing compounds is equal to 7. The dc magnetic susceptibility and isothermal magnetization have been measured in magnetic fields up to $0.43 \mathrm{~T}$ with a "Cahn 1000" balance magnetometer. The ac magnetic measurements have been carried out in the temperature range $4.2-300 \mathrm{~K}$ using a Lake Shore 7225 Model susceptometer by applying ac fields $1 \times 10^{-5}-2 \times 10^{-4} \mathrm{~T}$ and dc fields up to $5 \mathrm{~T}$. Some measurements have been performed on an Oxford Instruments magnetometer at temperatures of 1.5-50 $\mathrm{K}$ and on a SQUID magnetometer of an original design. It should be noted that ac and dc measurements have given the similar experimental values. The effective magnetic moment $\mu_{\text {eff }}$ and the paramagnetic Curie temperature $\Theta$ have been determined from the linear part of the temperature dependent inverse susceptibility curve $\chi^{-1}(T)$ in the temperature range from $77 \mathrm{~K}$ up to room temperature. The electrical resistivity has been measured using a standard four-probe technique.

\section{Results and discussion}

Depending on the rare-earth metal the 1212 compounds exhibit different magnetic properties. The 1212 compounds with non-magnetic $\mathrm{Y}$ and $\mathrm{Lu}$ ( $\mathrm{AlSr}_{2} \mathrm{YCu}_{2} \mathrm{O}_{7-x}, \mathrm{AlSr}_{2} \mathrm{LuCu}_{2} \mathrm{O}_{7-x}$ and $\mathrm{TlSr}_{2} \mathrm{LuCu}_{2} \mathrm{O}_{7-x}$ ) show a sharp peak on the susceptibility curves vs. temperature in the temperature range of $15-22 \mathrm{~K}$ (Fig. 1). This sharp peak shifts towards the low temperature with increasing magnetic field (see Fig. 1b). There is no hysteresis of the magnetic moment at the temperatures below the peak. This type of behaviour seems to be characteristic of quasi-two-dimensional antiferromagnetic ordering (AFM) of Cu-spins. Some other investigated compounds $\mathrm{AlSr}_{2} \mathrm{LnCu}_{2} \mathrm{O}_{7-x}, \mathrm{TlSr}_{2} \mathrm{LnCu}_{2} \mathrm{O}_{7-x}, \mathrm{TlBa}_{2} \mathrm{LnCu}_{2} \mathrm{O}_{7-x}$ with magnetic rare-earth ions ( $\mathrm{Ln}=\mathrm{Nd}, \mathrm{Gd}, \mathrm{Tb}, \mathrm{Dy}, \mathrm{Ho}$, and $\mathrm{Er}$ ) reveal quite different features in their magnetic characteristics. The thermomagnetic curves are described well by the Curie-Weiss law at low magnetic fields but in high magnetic fields we can see maxima that are displaced to the higher temperatures with increasing magnetic fields. Figure 2 shows a typical dependence $\chi(T)$ for $\mathrm{TlSr}_{2} \mathrm{GdCu}_{2} \mathrm{O}_{7-x}$ for different applied fields. No hysteresis on the magnetization curve $M(H)$ has been observed at the temperatures both above and below the maximum (see Fig. 2b). As the described features of $\chi(T)$ curve can be attributed to AFM ordering we can assume that a new type of antiferromagnetic ordering-field induced AFM has been found experimentally [2]. According to the theoretical model of Nagaev [3], the long-range antiferromagnetic order in two-dimensional 

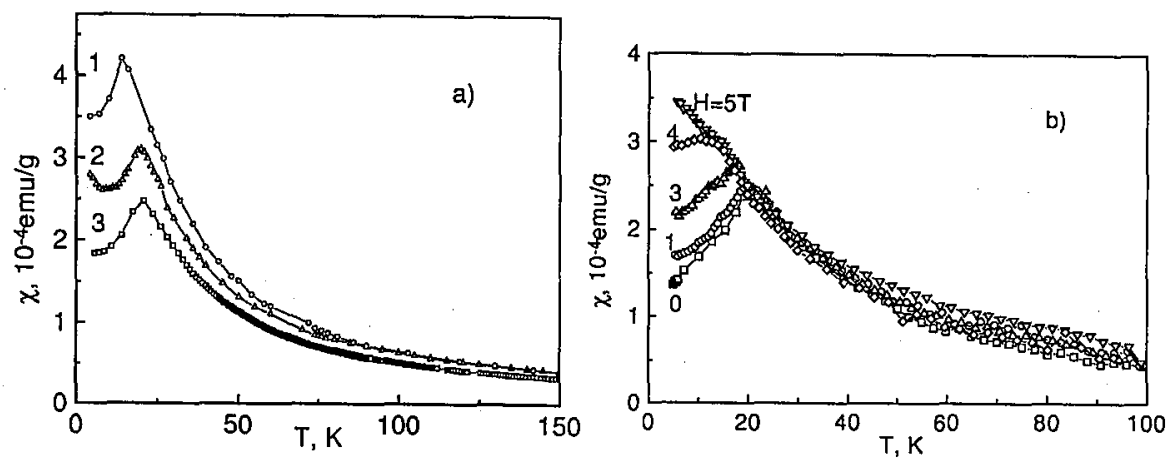

Fig. 1. (a) Temperature dependence of dc susceptibility for $\mathrm{AlSr}_{2} \mathrm{YCu}_{2} \mathrm{O}_{7-x}$ (1), $\mathrm{AlSr}_{2} \mathrm{LuCu}_{2} \mathrm{O}_{7-x}$ (2), and $\mathrm{TlSr}_{2} \mathrm{LuCu}_{2} \mathrm{O}_{7-x}$ (3) at $H=4.3$ Oe. (b) Temperature dependence of ac susceptibility for $\mathrm{TISr}_{2} \mathrm{LuCu}_{2} \mathrm{O}_{7-x}$ at $H$ from 0 to $5 \mathrm{~T}$.
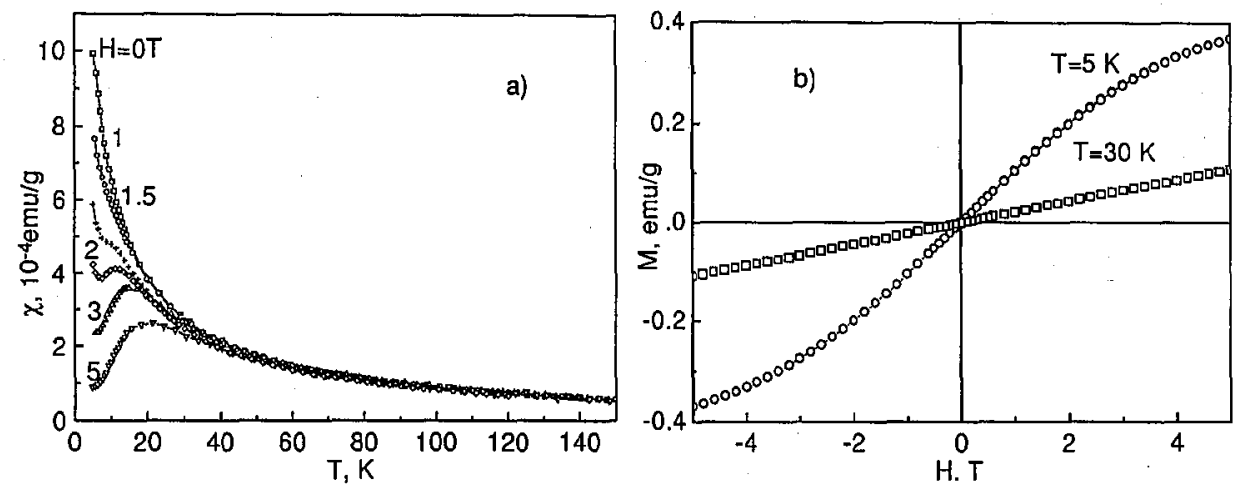

Fig. 2. (a) Temperature dependence of ac susceptibility for $\mathrm{TSR}_{2} \mathrm{GdCu}_{2} \mathrm{O}_{7-x}$ at different magnetic fields. (b) Dependence of magnetization of $\mathrm{TlSr}_{2} \mathrm{GdCu}_{2} \mathrm{O}_{7-x}$ at the temperatures $5 \mathrm{~K}$ and $30 \mathrm{~K}$.

spin-1/2 Heisenberg frustrated systems can be induced by external magnetic field because it decreases the angles between magnetic moments in the magnetic sublattice and suppresses quantum spin fluctuations hindering AFM ordering in such systems at $T=0$. The additional favourable factors might be the magnetic anisotropy, interplane exchange interaction, and interaction of $1 / 2$ spins with large spins of rare-earth metals [4]. Third group of studied magnetic compounds with 1212 structure includes $\mathrm{AlSr}_{2} \mathrm{TmCu}_{2} \mathrm{O}_{7-x}, \mathrm{TlSr}_{2} \mathrm{TmCu}_{2} \mathrm{O}_{7-x}$, and $\mathrm{TlBa}_{2} \mathrm{TmCu}_{2} \mathrm{O}_{7-x}$. At the temperature dependences of susceptibility one can see a sharp peak at $15-20 \mathrm{~K}$, which drastically drops with rather small (about $1 \mathrm{kOe}$ ) values of the external magnetic field (see Fig. 3a) for $\mathrm{TlSr}_{2} \mathrm{TmCu}_{2} \mathrm{O}_{7-x}$ ). The considerable hysteresis of magnetic moment at temperatures below transition clearly seen in Fig. 3b bear witness in favour of ferromagnetic-like ordering (FM). In order to study the interaction between the superconducting and the ferromagnetic order parameters we have investigated a set of solid solutions $\mathrm{TlBa}_{2}\left(\mathrm{Ca}_{1-y} \mathrm{Tm}_{y}\right) \mathrm{Cu}_{2} \mathrm{O}_{7-x}$ and $\mathrm{TlSr}_{2}\left(\mathrm{Ca}_{1-y} \mathrm{Tm}_{y}\right) \mathrm{Cu}_{2} \mathrm{O}_{7-x}$ with a wide range of properties from high- $T_{\mathrm{c}}$ super- 

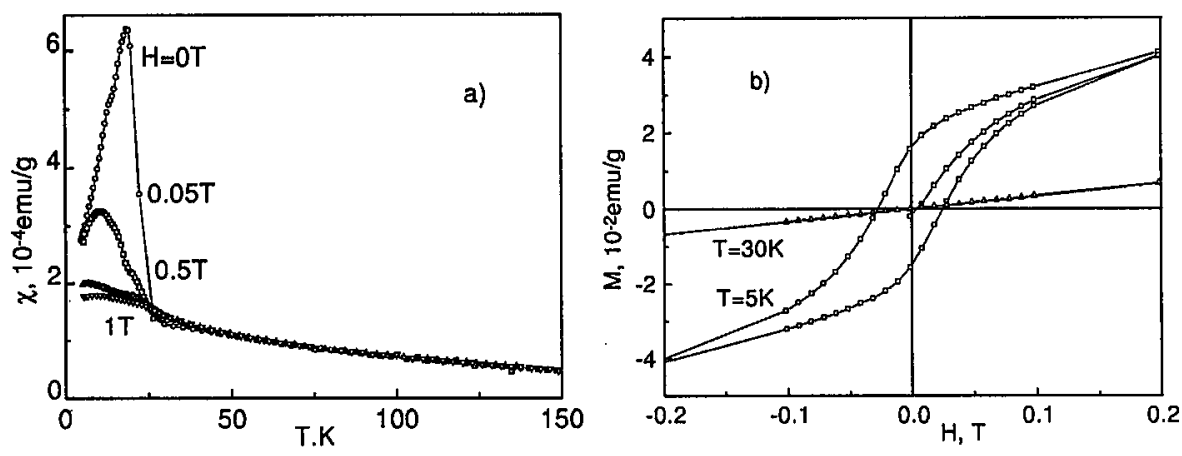

Fig. 3. (a) Temperature dependence of ac susceptibility for $\mathrm{TlSr}_{2} \mathrm{TmCu}_{2} \mathrm{O}_{7-x}$ at different magnetic fields. (b) Dependence of magnetization of $\mathrm{TISr}_{2} \mathrm{TmCu}_{2} \mathrm{O}_{7-x}$ at the temperatures 5 and $30 \mathrm{~K}$.

conducting $\mathrm{TlBa}_{2} \mathrm{CaCu}_{2} \mathrm{O}_{7-x}$ and $\mathrm{TlSr}_{2} \mathrm{CaCu}_{2} \mathrm{O}_{7-x}$ up to non-superconducting low-temperature ferromagnetic samples for $y=1$. The measurements indicate that up to $y=0.7$ the samples exhibit zero resistivity and the value of critical temperature does not vary considerably. But for $y=0.7$ from dc-magnetization data we can see that the samples become superconducting at $T_{\mathrm{c}}=100 \mathrm{~K}$ and exhibit the features similar to $\mathrm{FM}$ TlBa $2 \mathrm{TmCu}_{2} \mathrm{O}_{7-x}$ on the $\chi(T)$ dependence at a much lower temperature about $20 \mathrm{~K}$. So, one can conclude that in the system of these solid solutions superconducting and FM phases can exist simultaneously. The results of temperature-dependent magnetic moment measured by SQUID magnetometer for $\mathrm{TlBa}_{2}\left(\mathrm{Ca}_{0.2} \mathrm{Tm}_{0.8}\right) \mathrm{Cu}_{2} \mathrm{O}_{7-x}$ both in the case of zero-field-cooling and field-cooling indicate the behaviour typical of superconductors. On the other hand, on the base of magnetization data for $\mathrm{TlBa}_{2}\left(\mathrm{Ca}_{0.1} \mathrm{Tm}_{0.9}\right) \mathrm{Cu}_{2} \mathrm{O}_{7-x}$ we have found that superconductivity and ferromagnetic ordering coexist in the sample. In the samples of $\mathrm{TlSr}_{2} \mathrm{NdCu}_{2} \mathrm{O}_{7-x}$ and $\mathrm{TlBa}_{2} \mathrm{DyCu}_{2} \mathrm{O}_{7-x}$ the phase separation on the superconducting, paramagnetic insulating, and field-induced antiferromagnetic phases has been observed. So the compounds with the 1212-type of structure appear to demonstrate different types of long-range ordering, namely: superconducting and three types of magnetic ordering. Moreover, these phenomena may coexist in the same sample simultaneously. The work is supported by the grant RFFI-NNIO, project no. 96-02-00046G.

\section{References}

[1] V. Bobrovskii, A. Mirmelstein, A. Podlesnyak, E. Mitberg, E. Khlybov, P. Fischer, A. Furrer, Physica B 234-236, 862 (1997).

[2] E.P. Khlybov, I.E. Kostyleva, K. Nenkov, G. Fuchs, K.-H. Muller, T. Palewski, J. Warchulska, Phys. Met. Metallogr. 86, 31 (1998).

[3] A.V. Micheenkov, E.L. Nagaev, E.V. Zhasinas, Phys. Lett. A 205, 101 (1995).

[4] E.P. Khlybov, E.L. Nagaev, E.V. Zhasinas, K. Nenkov, G. Fuchs, Phys. Rev. B 56, 8075 (1997). 